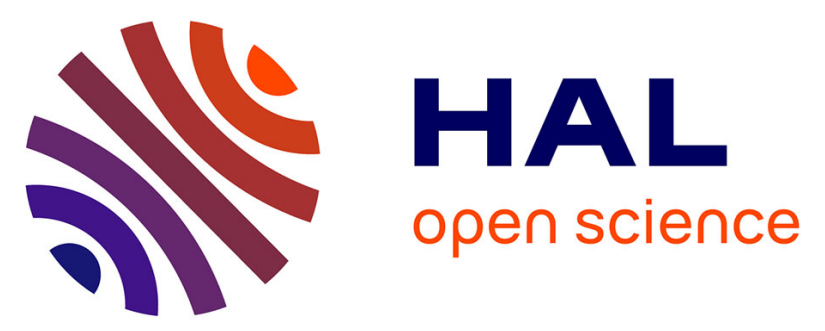

\title{
N -(2-Ethylhexyl)carbazole: A New Fluorophore Highly Suitable as a Monomolecular Liquid Scintillator
}

Eva Montbarbon, Fabien Sguerra, Guillaume H. V. Bertrand, Élodie Magnier, Romain Coulon, Robert B. Pansu, Matthieu Hamel

\section{- To cite this version:}

Eva Montbarbon, Fabien Sguerra, Guillaume H. V. Bertrand, Élodie Magnier, Romain Coulon, et al.. N -(2-Ethylhexyl)carbazole: A New Fluorophore Highly Suitable as a Monomolecular Liquid Scintillator. Chemistry - A European Journal, 2016, 22 (34), pp.12074-12080. 10.1002/chem.201601749 . hal-01390268

\section{HAL Id: hal-01390268 \\ https://hal.science/hal-01390268}

Submitted on 26 Mar 2020

HAL is a multi-disciplinary open access archive for the deposit and dissemination of scientific research documents, whether they are published or not. The documents may come from teaching and research institutions in France or abroad, or from public or private research centers.
L'archive ouverte pluridisciplinaire HAL, est destinée au dépôt et à la diffusion de documents scientifiques de niveau recherche, publiés ou non, émanant des établissements d'enseignement et de recherche français ou étrangers, des laboratoires publics ou privés. 


\title{
$\mathrm{N}$-(2-ethylhexyl)carbazole: a new fluorophore highly suitable as a monomolecular liquid scintillator
}

\author{
Eva Montbarbon, ${ }^{[a, b, c]}$ Fabien Sguerra, ${ }^{[a]}$ Guillaume H. V. Bertrand, ${ }^{[a]}$ Élodie Magnier, ${ }^{[a]}$ Romain \\ Coulon, ${ }^{[a]}$ Robert B. Pansu, ${ }^{[b]}$ and Matthieu Hamel ${ }^{*[a]}$
}

Abstract: In this work we report the synthesis, the photophysical properties and the applications in scintillation counting of $\mathrm{N}-(2$ ethylhexyl)carbazole $(\mathrm{EHCz})$. This molecule displays all required characteristics for an efficient liquid scintillator (emission wavelength, scintillation yield), and can be used without any extra fluorophores. Thus, its scintillation properties are discussed, as well as its fast neutron/gamma discrimination. For the latter application, the material is compared with traditional liquid scintillator BC-501A, and other liquid fluorescent molecules classically used as scintillation solvents such as xylene, pseudocumene (PC), linear alkyl benzene (LAB), diisopropylnaphthalene (DIN), 1-methylnaphthalene (1-MeNapht) and 4-isopropylbiphenyl (iPrBiph). For the first time, an excimeric form of a molecule is advantageously used in scintillation counting. A moderate discrimination between fast neutrons from gamma rays was observed in bulk $\mathrm{EHCz}$, with an apparent neutron/gamma discrimination potential that is one half of BC-501A.

\section{Introduction}

Liquid scintillators are mostly ternary mixtures of two different fluorophores dissolved in an aromatic solvent. When a radiation impinges the material, it deposits part (Compton scattering) or totality (photoelectric effect) of its energy. At the end of the ensuing ionisation cascade and via Förster energy transfer the scintillator gives a luminescent response preferentially in the visible spectrum, ideally centered around $420 \mathrm{~nm}$, where most of the commercial photomultiplier tubes have an optimal quantum efficiency. Such sensors find various applications, from nuclear research to high energy physics. The most known liquid scintillator is perhaps Saint-Gobain's BC-501A (and its EJ-301 analogue from Eljen Technology), which is a xylene-based liquid scintillator, characterized by a $425 \mathrm{~nm}$ emission, a fast photoluminescence decay time, a high light yield $\left(78 \%\right.$ of anthracene, i.e. $\approx 12,000$ ph. $\mathrm{MeV}^{-1}$ ) and an efficient Pulse Shape Discrimination (PSD) capability

[a] E. Montbarbon, Dr. F. Sguerra, Dr. G. H. V. Bertrand, É. Magnier, Dr. R. Coulon, Dr. M. Hamel

CEA, LIST, Laboratoire Capteurs \& Architectures Electroniques CEA Saclay, 91191 Gif-sur-Yvette cedex (France)

E-mail: matthieu.hamel@cea.fr

[b] E. Montbarbon, Dr. R. B. Pansu

Laboratoire de Photophysique et Photochimie Supramoléculaires et Macromoléculaires (CNRS UMR 8531), École Normale Supérieure de Cachan, 61 Avenue du Président Wilson, F-94235 Cachan cedex, France.

[c] Doctoral School INTERFACES, Paris-Saclay University, Saclay, France.

Supporting information for this article is given via a link at the end of the document. between fast neutrons and gamma rays.

The last decade has seen many efforts in the chemical improvement of organic scintillators, both in the liquid ${ }^{[1]}$ or the plastic $^{[2]}$ states. To the physicist concern, liquid scintillators present several drawbacks, as they are prepared from hazardous solvents with low flashpoint and must be sealed under inert atmosphere to retain their properties. Most of the recent improvements are therefore performed in the frame of better liquid scintillators for PSD purpose, but also for a reduced toxicity, biodegradation and not-flammability.

As early as 1958, scientists were already concerned about flammability and volatility of liquid scintillators, and metaand para-isopropylbiphenyl's isomers were the first molecules considered as a decent alternative. ${ }^{[3]}$ But it was only in the 2000's that chemical tuning provided EJ-309 liquid scintillator, designed for PSD, and composed of an almost not-flammable solvent (most probably phenylxylylethane, PXE). Pseudocumene is the base of Pico-Fluor 40, and LAB and DIN are found now in many liquid scintillators. In the frame of $n / y$ pulse shape discrimination, it was recently reported, for the first time, a moderate PSD in non-aromatic solvents, such as ethanol or heptane. ${ }^{[4]}$ Non-hydrocarbon solvents are also extensively studied. ${ }^{[5]}$ Ultimately, scintillating systems were also almost reached in water. ${ }^{[6]}$

In this context, we wish to present herein our most recent work concerning new liquid scintillators. More precisely, we would like to introduce the use of $\mathrm{N}$-(2-ethylhexyl)carbazole $(\mathrm{EHCz})$, a liquid fluorescent molecule, that can act as a whole liquid scintillator. It does not need indeed to be loaded with UV and violet-emitting fluorophores for scintillation purpose.

To the best of our knowledge, there is no example in the literature of liquid scintillator made of a unique compound acting as both solvent and emitter, unless traditional solvents used to prepare liquid scintillators (e.g. xylene, toluene, benzene) were considered as such. We wish to introduce herein $\mathrm{N}$-(2-ethylhexyl)carbazole (hereafter designated as $\mathrm{EHCz}$ ) as a potent one component liquid scintillator, moreover being used at its excimer form. This molecule is already known in the field of liquid dye laser, ${ }^{[7]}$ as very recently $\mathrm{EHCz}$ was used as solvent-free liquid organic laser for Distributed Feedback application. ${ }^{\left[{ }^{[3}\right.}$ A multi-gram synthesis affords an easy access to the molecule, which we fully describe in terms of photophysical properties. Then the main characteristics of scintillation counting are given and the pulse shape discrimination 
between fast neutrons and gamma rays is ultimately evaluated.

Table 1. Main characteristics of suitable solvents for the preparation of liquid scintillators.

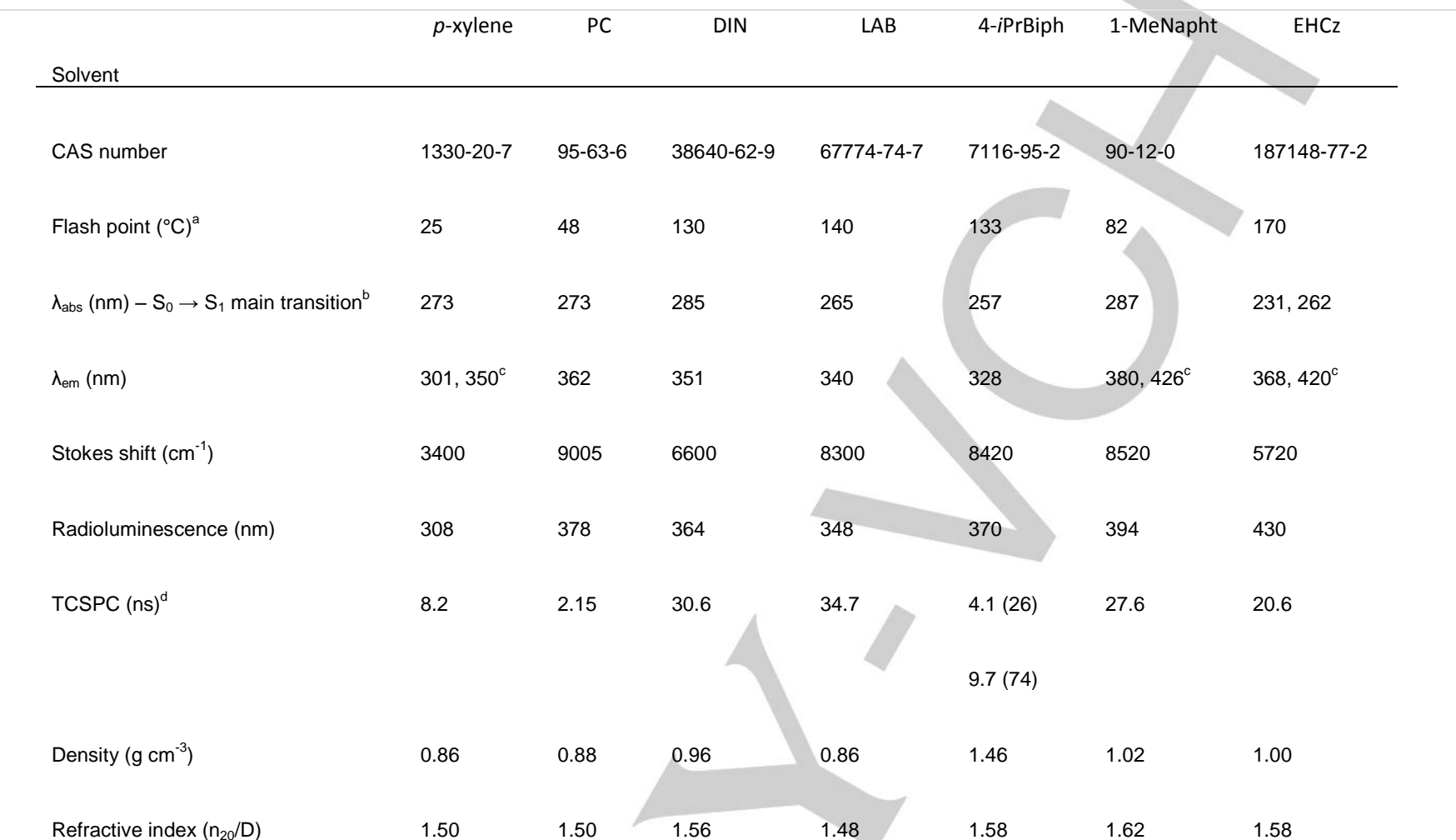

${ }^{\mathrm{a}}$ closed cup; ${ }^{\mathrm{b}}$ concentration $10^{-2}-10^{-4} \mathrm{~mol} \mathrm{~L}^{-1}$ in spectroscopic cyclohexane; ${ }^{\mathrm{C}}$ emission wavelength of the excimer; ${ }^{\mathrm{d}}$ considered as a monoexponential decay, unless otherwise stated; data relative to the bulk liquid.

\section{Results and Discussion}

\section{State of the art}

Table 1 resumes relevant characteristics of regular solvents used in liquid scintillation: xylene(s) - mainly its para isomer, pseudocumene (PC), diisopropylnaphthalene (in the form of multiple isomers, DIN), linear alkyl benzene (a complex mixture of organic compounds with formula $C_{6} H_{5} C_{n} H_{2 n+1}$, with $n$ in the range 10 to $16, L A B)$, 4-isopropylbiphenyl (4-iPrBiph) and 1 methylnaphthalene (1-MeNapht). These solvent usually are methylated derivatives of benzene, naphthalene or biphenyl (Figure 1). Density, flash point and refractive index are from the literature, when available, or from experiments performed in our institute.

Unfortunately, most of these solvents have major drawbacks and cannot be used without addition of one or many fluorophores: (1) they have a rather low flashpoint; (2) can be hazardous when handled; or (3) the radioluminescence maximum is under $400 \mathrm{~nm}$ (photomultiplier tubes used for scintillation purpose have a maximal quantum efficiency between 400 and $450 \mathrm{~nm}$ and thus radiation detection is less efficient for fluorophores emitting bellow $400 \mathrm{~nm}$ ).

Probably the main drawback associated with liquid scintillators is their low flash points, which prevent them from several applications, at the benefit for plastic scintillators. For instance, these last years have seen several developments for replacing liquid scintillators by plastics in the field of neutron/gamma discrimination. ${ }^{[9]}$ Table 1 references data from the literature (usually the MSDSs), except the value for $\mathrm{EHCz}$ which was experimentally determined. It shows that only 4 out of 7 common solvents have a flash point in the range $100-150^{\circ} \mathrm{C}$, and xylene(s) and pseudocumene have a very limited flash point (25 and $48^{\circ} \mathrm{C}$, respectively). In this context, only liquid siloxanes are competitive, but still need the loading of primary and secondary fluorophores, ${ }^{[5]}$ thus leading to probable loss of light if the Förster energy transfer is not sufficient enough. In that context $\mathrm{EHCz}$ could be an alternative because of its high flashpoint $\left(170^{\circ} \mathrm{C}\right)$ and an intense radioluminescence centered at $420 \mathrm{~nm}$, all these advantages being obtained with this single molecule, at its excimer form.

\section{Synthesis}

A thorough literature survey reveals that a carbazole substituted at the $N$ position with a hydrocarbon, alkyl moiety always almost leads to a solid molecule at room temperature. The two known exceptions are $\mathrm{EHCz}$, whose melting point is close to $0^{\circ} \mathrm{C}$, and a poly(ethylene oxide) with carbazole moiety. ${ }^{[10]}$ The latter compound displays a slightly lower photoluminescence quantum yield (0.28) compared with $\mathrm{EHCz}$ (0.31). Although $\mathrm{EHCz}$ is a commercial product, it is rather expensive when large volumes are needed. We therefore turned our attention into a straightforward synthesis, involving the reaction between carbazole and 2-ethylhexyl bromide in the presence of sodium hydride (Scheme 1). The titled compound was isolated as a colourless, viscous liquid after chromatography on silica gel and/or 
distillation. The optimized sequence allowed us preparing the titled compound on a multi-gram scale. As expected for scale up the yield was decreased, also minimally, from $82 \%$ for a $5 \mathrm{~g}$ synthesis down to $73 \%$ for a $25 \mathrm{~g}$ preparation. Purification by flash chromatography on silica gel gave us a purity suitable enough for scintillation application (scintillation grade).

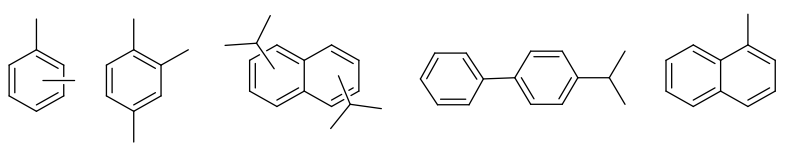

Figure 1. Topological representation of xylene(s), pseudocumene, diisopropylnaphthalene (mixture of isomers), 4-isopropylbiphenyl, and 1methylnaphthalene (from left to right). LAB is not shown due to the complex composition. BC-501A is a xylene-based liquid scintillator containing various fluorophores.<smiles>c1ccc2c(c1)[nH]c1ccccc12</smiles>

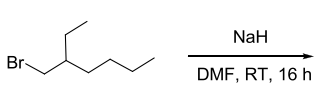<smiles>CCCCC(CC)Cn1c2ccccc2c2ccccc21</smiles>

yield $82 \%$

Scheme 1. Synthesis of $N$-(2-ethylhexyl)carbazole.

\section{Photophysical characterization}

Fluorescence spectra, radioluminescence, Time Correlated Single Photon Counting (TCSPC) and quantum yields were recorded in our facility (see Electronic supplementary information for experimental details). Density and refractive indexes could be found in the literature. However, we recorded $\mathrm{EHCz}$ refractive index and found a value of 1.58 at $589 \mathrm{~nm}$, and a higher value of 1.64 at $404 \mathrm{~nm}$. The refractive indexes from 404 to $706 \mathrm{~nm}$ are given in the supplementary material, with a relative standard uncertainty of \pm 0.001 .

Pure EHCz displays a structured emission with two peaks at 417 and $434 \mathrm{~nm}$ (Figure 2, top). Fluorescence lifetime was acquired by time correlated single photon counting (TCSPC) and was found to be $20 \mathrm{~ns}$. Once diluted in dichloromethane $\left(10^{-2}\right.$ to $10^{-4}$ $\mathrm{mol} \mathrm{L}^{-1}$ ) the emission is still structured and blue-shifted to 367 and $351 \mathrm{~nm}$, respectively, and the emission lifetime is shortened to 10.2 , then $6.3 \mathrm{~ns}$. The hypsochromic shifts, accentuated by the dilution, are consistent with a decrease in the number of molecules participating in the excimer. At low concentration the shift stops and the emission bands present a thinner structure, characteristic of mono-molecular excited species. This behavior is typical of carbazole emitting as a single species, and emission wavelength dependence with the concentration of $\mathrm{EHCz}$ was already reported by Kubota and coworkers. ${ }^{[10]}$ The same conclusion can be drawn from the time resolved experiments (Figure 2, bottom). The slower time response from pure $\mathrm{EHCz}$ can be attributed to high order excimer, where excited states delocalization increases its stability, whereas high dilution provides faster decay time corresponding to less delocalized excited state and ultimately monomolecular exciton. These photophysical properties are characteristics of carbazole derivatives excimers that can be seen for instance in polyvinylcarbazole (lifetime of about $42 \mathrm{~ns}$ at $420 \mathrm{~nm}^{[11]}$ ). Time measurements of other solvents reported in the Table 1 are also given in the Supporting Information (Figure SI1).
When diluted, the Stokes shift of EHCz is close to $5,700 \mathrm{~cm}^{-1}$ (based on the third shoulder at $293 \mathrm{~nm}$ at the concentration 10 ${ }^{4} \mathrm{M}$, see Table 2). Compared with other solvents, this is not the highest Stokes shift ever recorded, as Pseudocumene (PC) presents a $9,000 \mathrm{~cm}^{-1}$ value. To determine the Stokes shift of the neat form, the absorption spectrum was recorded using a $0.05 \mathrm{~mm}$ long optical cell. The last absorption shoulder was seen at $386 \mathrm{~nm}$, giving therefore a reduced Stokes shift of about $3,900 \mathrm{~cm}^{-1}$ (for both calculations the radioluminescence maximum was taken into account). However the transparency of the liquid was good enough at its emission wavelength, with a $78 \%$ value at $420 \mathrm{~nm}$.

Fluorescence quantum yield of $\mathrm{EHCz}$ was calculated in three dichloromethane-based solutions (ranging from 0.2 to $0.910^{-4}$ $\mathrm{mol} \mathrm{L}^{-1}$ ) and was evaluated to be $0.70 \pm 0.02$ which is way higher than the other scintillation solvents cited above (see Table 1). The main photophysical characteristics of $\mathrm{EHCz}$ diluted in dichloromethane or in neat form are given in Table 2.
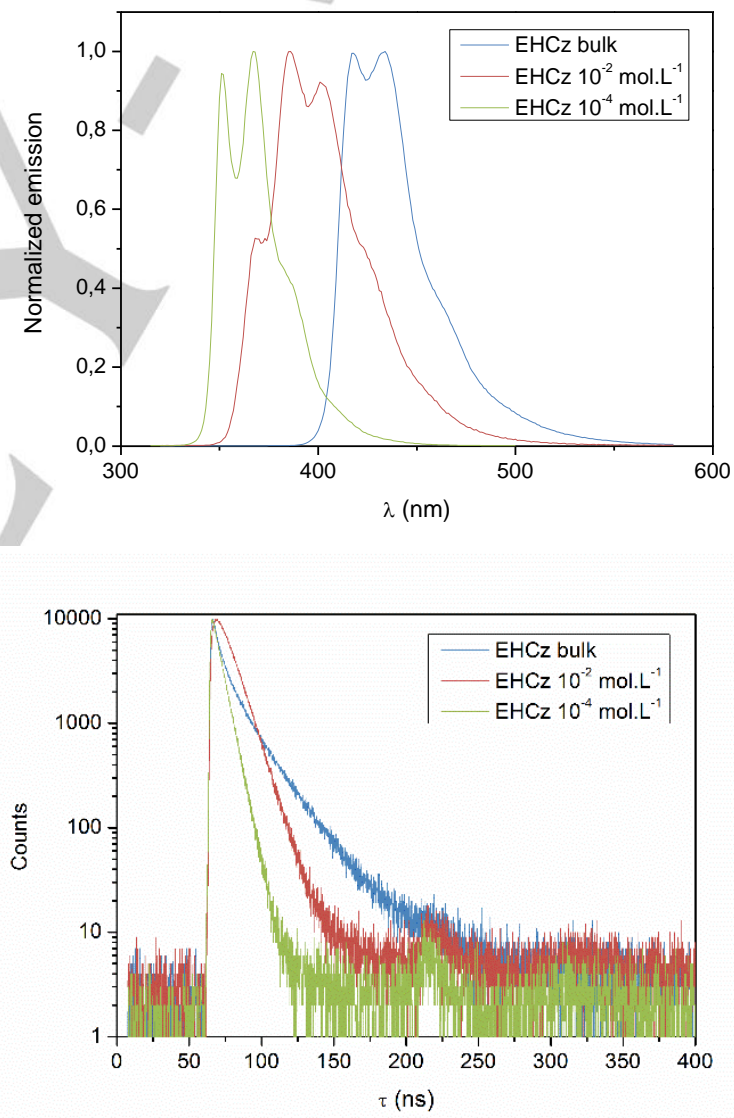

Figure 2. Influence of the $\mathrm{EHCz}$ concentration on fluorescence spectra (top) and fluorescence decay times (bottom). For the TCSPC experiment: $\lambda_{\mathrm{ex}}=$ $274 \mathrm{~nm} ; \lambda_{\text {monitoring }}$ at their respective maximum of emission.

\section{Radioluminescence}

Using the Fluoromax-4P spectrofluorimeter with the excitation light shut down, and a highly active ${ }^{90} \mathrm{Sr}^{90} \mathrm{Y}$ beta emitting radioactive source $\left(E_{\text {mean }}=933 \mathrm{keV}, E_{\max }=2280\right.$ $\mathrm{keV})$, we were able to record the optical response of liquid 
scintillators. To this, the emission slit was opened at its maximum available on this device, which presents the drawback to enlarge the emission response, leading therefore to pseudo-Gaussian shapes instead of welldefined luminescence spectra. Under ionizing radiation, $\mathrm{EHCz}$ displays a broad emission spectrum centered on $430 \mathrm{~nm}$ (Figure 3) and a FWHM of $57 \mathrm{~nm}$ which is similar to $\mathrm{BC}-501 \mathrm{~A}$ (emission at $428 \mathrm{~nm}$ and FWHM of $60 \mathrm{~nm}$ ). It is also noteworthy that $\mathrm{EHCz}$ is, after $\mathrm{BC}-501 \mathrm{~A}$, the best emitter of scintillation photons. The number of scintillation photons emitted during the time experiment is indexed for each liquid scintillator in Table 3.

Table 2. Photophysical properties of $\mathrm{N}$-(2-ethylhexyl)carbazole at various concentrations.

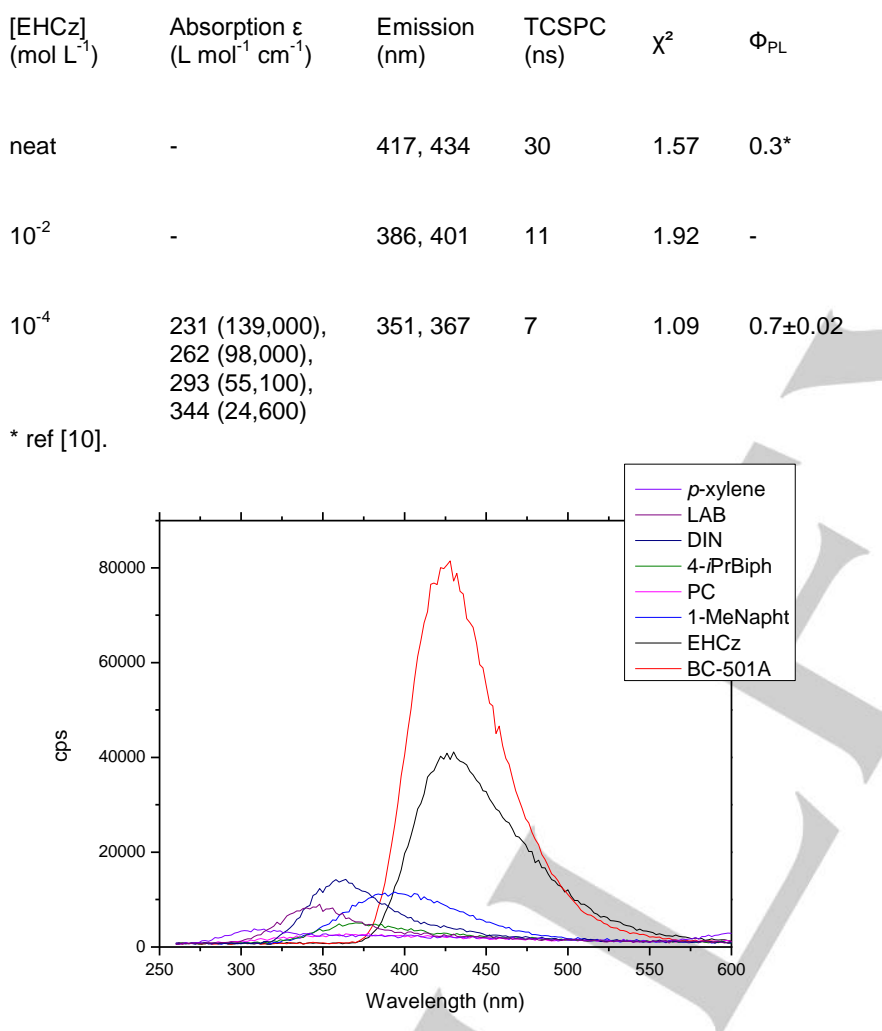

Figure 3. Radioluminescence spectra of pure liquids under $\beta$ irradiation $\left({ }^{90} \mathrm{Sr}\right.$ ${ }^{90} \mathrm{Y}-36 \mathrm{MBq}$ ): $p$-xylene, LAB, DIN, 4-isopropylbiphenyl, pseudocumene, 1methylnaphthalene, BC-501A, and EHCz (from lower to higher wavelengths of maximum emission).

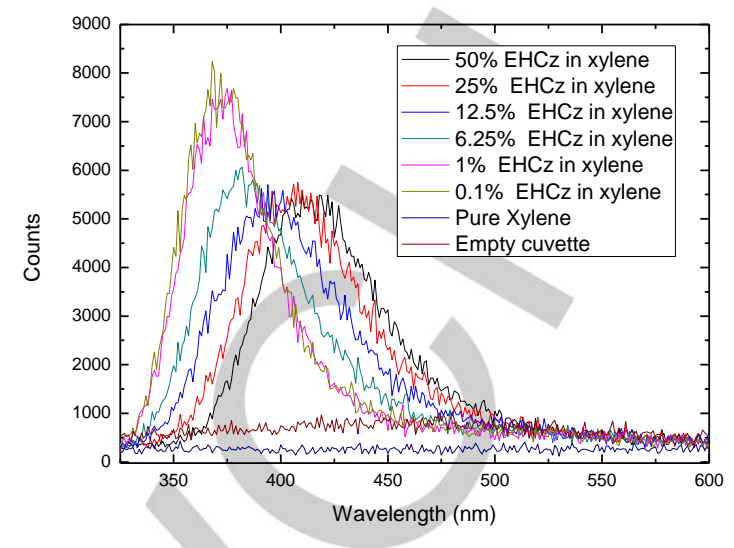

Figure 4. Radioluminescence spectra under $\beta$ irradiation $\left({ }^{90} \mathrm{Sr}^{90} \mathrm{Y}-25 \mathrm{MBq}\right)$ for $0-50$ vol\% EHCz in xylene. No emission of xylene is visible in this experiment.

In order to understand the possible effect of excimer formation on the scintillation process, the luminescent response to the ${ }^{90} \mathrm{Sr}^{-90} \mathrm{Y}$ radioactive source was then recorded with the concentration of $\mathrm{EHCz}$ in xylene, from 0.1 up to 50 vol\% (Figure 4). P-xylene was used to promote the energy deposition of ionizing particles at high dilution. Upon dilution of $\mathrm{EHCz}$, the radioluminescence is blue shifted from 430 to $370 \mathrm{~nm}$. This hypsochromic shift correlated with the dilution is consistent with results from fluorescence measurements. At high concentration the broad radioemission band is due to an excimer or, less likely, an exciplex with the xylene, lower concentration gave a thinner band, once again characteristic of a low order excimer, or even monomolecular exciton. Integral beneath the radioluminescence curves shows that the concentration does not impact photon intensity, which means that excimer formation does not affect the scintillation process (see Figure $\mathrm{SI} 2$ in the supporting information). This result confirms that it is favorable to use pure $\mathrm{EHCz}$ as its emission wavelength is better suited for standard photomultiplier tubes and its efficiency is not affected by excimer formation. The dilution effect is also confirmed to be harmful when recording the energy spectra of a $37 \mathrm{MBq}$, ${ }^{137} \mathrm{Cs}$ radioactive source emitting $662 \mathrm{keV}$ gamma rays. Relative to pure $\mathrm{EHCz}, 50$ and 25 vol\% xylene solutions emitted 93 and $89 \%$ photons, with a total number of counts of 72 and $64 \%$, respectively. This is shown in the Figure SI3 in the supporting information.

$\mathrm{EHCz}$ has a strong stability towards irradiation. Radioluminescence monitored at $420 \mathrm{~nm}$ and performed during $30 \mathrm{~min}$ with a highly active $36 \mathrm{MBq}{ }^{90} \mathrm{Sr}^{-}{ }^{90} \mathrm{Y}$ beta source in close contact with the quartz cuvette did not display any kind of signal loss, as well as for BC-501A (see Figure SI4 in the supporting information). 


\section{Effective quantum efficiency for the detection of $\mathrm{EHCz}$}

$\mathrm{EHCz}$ was fully described in terms of its photophysical properties, this means for its intrinsic properties.

The intended application, which is neutron/gamma discrimination, involves a detection system, made of a photodetector and an electronic digitizer. The photodetector used at the laboratory is a Hamamatsu H1949-51 photomultiplier tube (PMT), designed from a standard bialkali photocathode but slightly shifted to the UV region compared with other standard PMTs.

In order to characterize the coupling between the scintillator and the photodetector, the quantum efficiency criterion is introduced. Effective quantum efficiency is the probability that a scintillation photon (created by interaction of radiation with matter) arriving at the photocathode of the PMT produces a photoelectron. ${ }^{[12]}$ Equation 1 gives the definition of effective quantum efficiency $\eta_{Q}$ of a given scintillator.

$\eta_{Q}=\frac{\int_{0}^{\infty} I(\lambda) Q(\lambda) d \lambda}{\int_{0}^{\infty} I(\lambda) d \lambda}$

Equation 1

Where $\lambda$ is the wavelength and $Q$ is the distribution of the photocathode efficiency (given by the PMT manufacturer, herein Hamamatsu).

Effective quantum efficiency values for tested solvents and BC-501A are listed in Table 3. According to Table 3, the coupling between EHCz and H1949-51 PMT is not the best, but its value is similar to that one of BC-501A. In fact, the weak difference between them is certainly due to the slight shift of the photocathode efficiency in the UV region.

Table 3. Quantum efficiency of various liquid dyes and BC-501A.

$\begin{array}{lcc}\text { Solvent } & N_{p h} & \eta_{Q}(\%) \\ \text { p-xylene } & & \\ & 339480 & 18.38 \\ \text { Pseudocumene } & 274360 & 19.30 \\ & & \\ \text { DIN } & 612870 & 22.49 \\ & & \\ & & \\ \text { LAB } & 439320 & 21.11 \\ & & \\ \text { 4-isopropylbiphenyl } & 340130 & 20.65 \\ \text { 1-methylnaphthalene } & 631930 & 22.34 \\ \text { EHCz } & 1746140 & 20.99 \\ \text { BC-501A } & 2837630 & 22.26 \\ & & \end{array}$

\section{Radiation detection}

Despite all the above mentioned qualities of $\mathrm{EHCz}$, the most important part to our eyes is the possibility to detect radiations. To this, $5 \mathrm{~cm}$ long quartz cuvettes were exposed to gamma rays. One of the edges of the cuvette is optically coupled with Rhodorsil optical grease to a standard photomultiplier tube (see experimental section for details). With a digital oscilloscope, each scintillating pulse is digitalized and then recorded. Then a histogram is built from the integration of the pulse area.

The spectra represented in Figure 5 are typical from a Compton interaction between the incident gamma ray and the scintillator; no full energy peak is thus observed. However, with the same experimental conditions, it is possible to evaluate the scintillation yield (expressed in photons per deposited $\mathrm{MeV}$ ), based on a rule of thumb with a known scintillator. In our case, it is BC-501A, with a 12,000 ph $\mathrm{MeV}^{-1}$. Assuming that the $\mathrm{EHCz}$ Compton edge is located at $50 \%$ of BC501-A's Compton edge, we can therefore determine that $\mathrm{EHCz}$ gives $6,000 \mathrm{ph} \mathrm{MeV}^{-1}$. From the radioluminescence spectra, $\mathrm{EHCz}$ liberates approximately $60 \%$ of photons relative to BC-501A, and it is the same percentage when comparing the Compton edges of $\mathrm{EHCz}$ and BC-501A.

\section{Neutron/gamma pulse shape discrimination}

One of the main advantages of liquid scintillators is their ability to perform a very good discrimination between fast neutrons and gamma rays. Again, appropriate solutions towards flammable scintillators are very welcome in this field. ${ }^{[4,13,14]}$ In this context, a $3 \mathrm{~cm}^{3}$ quartz cuvette was exposed to a $1 \mathrm{MBq},{ }^{252} \mathrm{Cf}$ neutron/gamma radioactive source, and its response was compared to a BC-501A sample of same dimension. Both results are presented in Figure 6. It shows that $\mathrm{EHCz}$ displays a moderate pulse shape discrimination in neat form, with a naked eye lecture giving neutrons and gamma lobes (in the top and bottom parts of the Figure, respectively). The separation is two times better for BC-501A, despite non-optimized digitizer parameters for the latter. Indeed, due to the large decay time difference between $\mathrm{EHCz}$ and $\mathrm{BC}-501 \mathrm{~A}$ liquid scintillators (20 and $3.2 \mathrm{~ns}$, respectively) and for the sake of a better comparison, digitizer parameters were kept equivalent for both experiments. It is noteworthy that a special care must be taken in the removal of any residual oxygen in the medium, which can preclude any PSD. In addition, 1-Methylnaphthalene and 4-iPrBiphenyl in their pure forms were also confronted to an AmBe source as well as with BC-501A, with another setup; they did not display any kind of discrimination as such (see Figures SI5-7 in the supporting information).

\section{Discussion}

To the best of our knowledge, this is the first time that excimers are advantageously used for the production of efficient liquid scintillators. The production of such excimers dissipates energy: typically $0.42 \mathrm{eV}$ in our case. But this 
loss of energy is at the benefit of both the Stokes shift and the suitability with photomultiplier tube quantum efficiency. This explains why $\mathrm{EHCz}$ does not require any extra fluorophore to be efficient enough. Compared with xylene and 1-methylnaphthalene which are also able to produce excimers when undiluted, at 349 and $428 \mathrm{~nm}$ wavelengths, respectively, $\mathrm{EHCz}$ is the best excimer liquid scintillator. Ultimately, it is always assumed that the energy occurring from radiation/matter interaction is transferred from the matrix to the fluorophore via Förster interaction. As herein this liquid scintillator is composed of only one molecule, this is therefore the first example of liquid scintillation counting without Förster interaction.

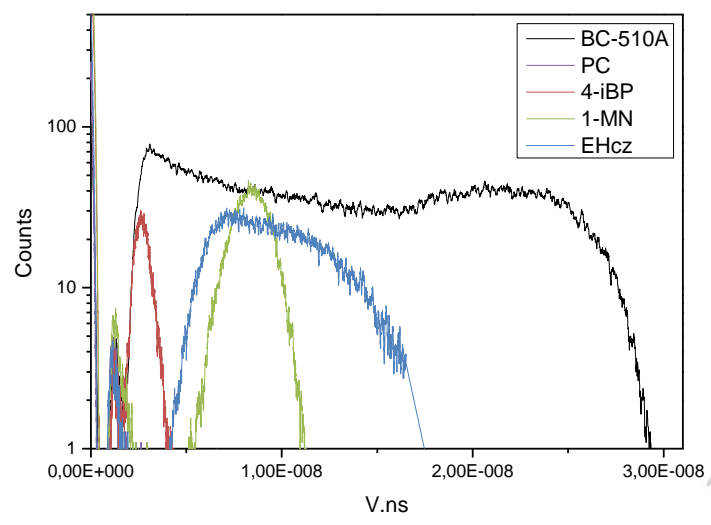

Figure 5. Comparison of the ${ }^{137} \mathrm{Cs}$ scintillation gamma spectra obtained for BC-501A (black), N-(2-ethylhexyl)carbazole (blue), 1 methylnaphthalene (green), 4-isopropylbiphenyl (red), where the background residual signal has been discarded. Data were obtained for $3 \mathrm{~mL}$ liquid samples.

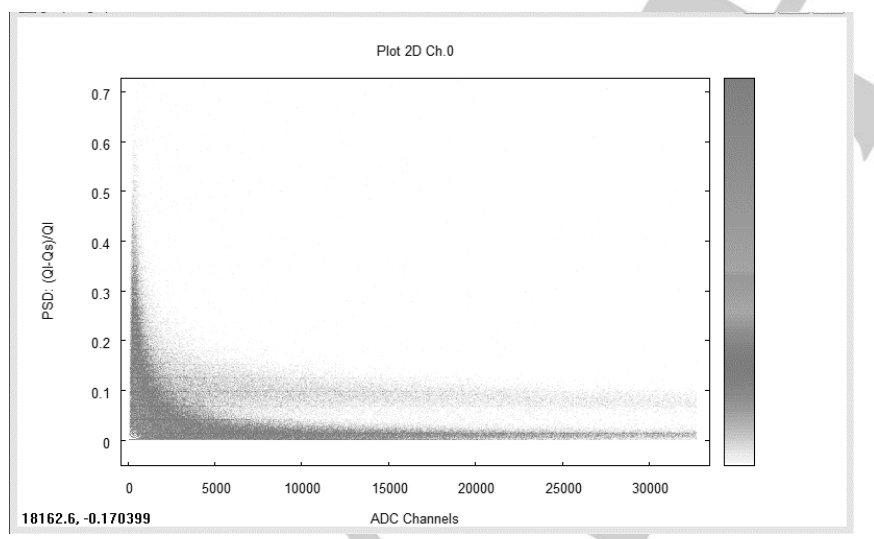

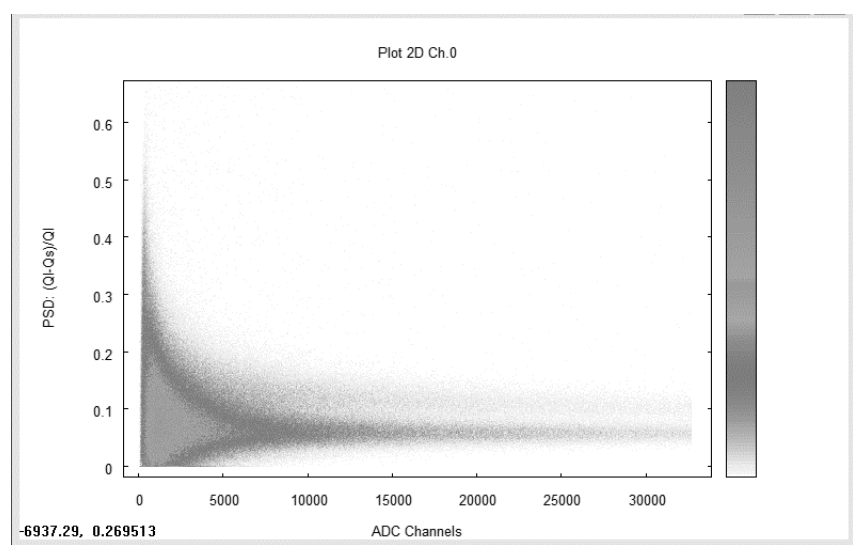

Figure 6. PSD plots for a ${ }^{252} \mathrm{Cf}$ radioactive source measurement with BC-501A (top) and EHCz (bottom). The same parameters for both samples were applied.

\section{Conclusions}

We report herein the use of $\mathrm{N}$-(2-ethylhexyl)carbazole, a colourless, fluorescent liquid molecule which can act as a liquid scintillator medium just by itself. It was prepared in high yield by deprotonation of carbazole followed by nucleophilic substitution with 2-ethylhexyl bromide. It is noteworthy that this reaction sequence can be extended to a 0.1 mole scale. No additive is required as the photophysical properties satisfy scintillation criteria. This compound was evaluated in terms of absorption and emission spectra, TCSPC, quantum yield and scintillation properties: decay time, energy spectra, and fast neutron/gamma pulse shape discrimination. As such, it respects all the criteria of a liquid scintillating system. It is optically transparent to its own light with a wavelength cut of the absorption around $402 \mathrm{~nm}$ for $1 \mathrm{~cm}$ optical path and absorbance 1. Both photoluminescence and scintillation wavelength maxima are located around $420 \mathrm{~nm}$, with the main emission driven by the excimers when used in bulk. The decay time is fast enough for scintillation application, and good scintillation yield was observed. Ultimately, we were glad to observe a visible pulse shape discrimination between fast neutrons and gamma rays.

At this stage we do not have any feedback regarding neither the chemical stability, nor its behaviour under high ionizing radiation flux, but it will be studied in future experiments.

\section{Acknowledgements}

C. Bobin is acknowledged for the loan of the refractometer. Perkin Elmer is acknowledged for the grant of DIN. G. Bélier is acknowledged for the access to the DIF facility. The authors thank the "Agence Nationale de la Recherche", within the CSOSG program (ANR-12-SECU-0002), and the 
French Direction Générale de l'Armement and Secrétariat Général de la Défense et de la Sécurité Nationale for their funding (G.H.V.B., F.S.).

\section{Experimental Section}

Xylene was obtained from VWR. 1-methylnaphthalene was obtained from Merck. These two solvents were used without purification. Pseudocumene was obtained from Sigma-Aldrich and was distilled prior to use. 4isopropylbiphenyl was obtained from Alfa Aesar and was purified on a silica gel pad prior to use. BC-501A was obtained from Saint-Gobain Crystals and Detectors. Sodium hydride and 2-ethylhexylbromide were obtained from Sigma Aldrich. Carbazole was purchased from Labosi or from Sigma-Aldrich.

${ }^{1} \mathrm{H}$ spectrum was recorded at $400 \mathrm{MHz}$ on a Bruker AVANCE 400 spectrometer. Chemical shifts $(\bar{\delta})$ are given in $\mathrm{ppm}$, referenced to the residual proton resonance of $\mathrm{CDCl}_{3}$ (7.26 ppm). Coupling constants $(\mathcal{J})$ are given in Hertz $(\mathrm{Hz})$. The terms $\mathrm{m}, \mathrm{d}$, $\mathrm{t}$, sept, refer to multiplet, doublet, triplet, septuplet.

EHCz was synthesized according to a previously published procedure. ${ }^{[15]}$ Carbazole (4 g, 23.9 mmol, 1 equiv.) and $\mathrm{NaH}$ (1.32 g, $55 \mathrm{mmol}, 2.3$ equiv., previously washed with pentane to get rid of mineral oil) were stirred under argon in DMF $(100 \mathrm{~mL})$ for $30 \mathrm{~min}$. 2-Ethylhexylbromide $(5.2 \mathrm{~mL}$, $29.3 \mathrm{mmol}, 1.2$ equiv.) was added dropwise and the mixture was stirred overnight. Water was added $(50 \mathrm{~mL})$ and the product was extracted with diethyl ether $(3 \times 100 \mathrm{~mL})$. The organic layer was concentrated to $50 \mathrm{~mL}$ and washed with brine $(3 \times 50 \mathrm{~mL})$. The organic layer was dried with anhydrous magnesium sulfate, filtered and evaporated. The product was purified by column chromatography $\left(\mathrm{SiO}_{2}\right.$ cyclohexane) to afford the product as colorless oil (5.5 g, 19.7 mmol, 82\%, Lit. $^{[15]} 49 \%$ ).

The same reaction was performed on a $20 \mathrm{~g}$ scale of carbazole, leading to $\mathrm{N}$-(2-ethylhexyl)carbazole with a $73 \%$ yield.

${ }^{1} \mathrm{H}$ NMR: $\delta 0.86$ (t, 3H, $\left.{ }^{3} J 7.3\right) ; 0.91$ (t, 3H, $\left.{ }^{3} J 7.3\right) ; 1.21-$ $1.43(\mathrm{~m}, 8 \mathrm{H}) ; 2.07$ (sept, $\left.1 \mathrm{H},{ }^{3} J 6.7\right) ; 4.10-4.21(\mathrm{~m}, 2 \mathrm{H})$ 7.39 (d, $4 \mathrm{H},{ }^{3}$ J 8.2); 7.45 (dt, $4 \mathrm{H},{ }^{3} J 6.9,{ }^{4}$ J 1.1$)$.

All photophysical experiments were conducted in Suprasil Quartz cuvettes.

UV-visible spectra were measured at room temperature in cyclohexane on a Jenway 6715 UV/Vis spectrometer, wavelengths are given in $\mathrm{nm}$ and extinction coefficients $\varepsilon$ are presented in $\mathrm{L} \mathrm{mol}^{-1} \mathrm{~cm}^{-1}$.

Fluorescence, radioluminescence and Time-Correlated Single Photon Counting (TCSPC) experiments were performed with a Horiba Jobin Yvon Fluoromax 4P spectrofluorometer. Radioluminescence spectra were acquired by using the following procedure. In the Fluoromax
4P spectrofluorometer, the excitation light was shut down. In the centre of the experimenter chamber, the ${ }^{90} \mathrm{Sr}^{9}{ }^{90} \mathrm{Y} \beta$ emitting source (25 or $37 \mathrm{MBq}$ ) placed close to the scintillator cell. Spectra were acquired with an integration time of $0.5 \mathrm{~s} \mathrm{~nm}^{-1}$. TCSPC experiments were performed by illuminating the liquid scintillator with a laser diode operating at $274 \mathrm{~nm}$.

Gamma spectra were recorded as follows: the sample was optically coupled to a Hamamatsu H1949-51 photomultiplier tube operating at negative voltage. A $387 \mathrm{kBq}{ }^{60} \mathrm{Co}$ radioactive source was used to excite the material. 100,000 pulses were recorded with a Lecroy HDO6054-MS digital oscilloscope, were integrated and sorted as an energy histogram. Dilution experiments were performed with a 37 $\mathrm{MBq}{ }^{137} \mathrm{Cs}$ radioactive source and pulses were sorted and treated with a custom-made electronic board. ${ }^{[16]}$

A Schmidt + Haensch DSR $\lambda$ refractometer was used for the determination of the $\mathrm{EHCz}$ refractive index.

Determination of the $\mathrm{EHCz}$ flash point was performed at Filab (www.filab.com).

Pulse shape discrimination leading to the separation of fast neutrons vs. gamma rays was performed using a CAEN DT5730B digitizer, monitored with the DPP-PSD software. An unshielded, $\approx 1 \mathrm{MBq}{ }^{252} \mathrm{Cf}$ source was placed $2 \mathrm{~cm}$ close to $3 \mathrm{~cm}^{3}$ samples. Experiments with other solvents were performed at CEA DAM/DIF using the FASTER module. ${ }^{[17]} 3 \mathrm{~cm}^{3}$ samples were coupled to a Hamamatsu H10580 PMT with optical grease, then exposed to an unshielded $370 \mathrm{MBq}$ AmBe source.

\section{Keywords: Carbazole $•$ Scintillation $•$ Fluorescence $・$ Excimer}

[1] M. F. L'Annunziata and M. J. Kessler, In "Handbook of Radioactivity Analysis (Third Edition)",Academic Press, 2012, pp. 423-573.

[2] G. H. V. Bertrand, M. Hamel and F. Sguerra, Chem. Eur. J., 2014, 20 15660-15685

[3] W. L. Buck and R. K. Swank, Rev. Sci. Instrum., 1958, 29, 252

[4] M. Hamel, A.-M. Frelin-Labalme and S. Normand, Europhys. Lett., 2014, 106, 52001

[5] M. Dalla Palma, S. M. Carturan, M. Degerlier, T. Marchi, M. Cinausero, F. Gramegna and A. Quaranta, Opt. Mater., 2015, 42, 111-117.

[6] M. Yeh, S. Hans, W. Beriguete, R. Rosero, L. Hu, R. L. Hahn, M. V. Diwan, D. E. Jaffe, S. H. Kettell and L. Littenberg, Nucl. Instr. Methods A, 2011, 660, 51-56.

[7] E. Y. Choi, L. Mager, T. T. Cham, K. D. Dorkenoo, A. Fort, J. W. Wu, A Barsella and J.-C. Ribierre, Opt. Express, 2013, 21, 11368-11375.

[8] J.-H. Kim, M. Inoue, L. Zhao, T. Komino, S. Seo, J.-C. Ribierre and C. Adachi, Appl. Phys. Lett., 2015, 106, 053302.

[9] G. H. V. Bertrand, M. Hamel, S. Normand and F. Sguerra, Nucl. Instr. Methods A, 2015, 776, 114-128.

[10] K. Kubota, S. Hirata, Y. Shibano, O. Hirata, M. Yahiro and C. Adachi, Chem. Lett., 2012, 41, 934-936.

[11] H. S. Nalwa, In "Advanced Functional Molecules \& Polymers Volume Three: Volume 3: Electronic and Photonic Properties", CRC Press, $2001, \mathrm{p} 8$.

[12] M. Miyajima, S. Sasaki and H. Tawara, IEEE Trans. Nucl. Sci., 1993 , 40, 417-423

[13] (a) M. Hamel, A.-M. Frelin-Labalme, V. Simic and S. Normand, Nucl. Instr. Methods A, 2009, 602, 425-431; (b) M. Hamel, S. Normand, A.-M. Frelin and V. Simic, LSC 2008, Advances in Liquid Scintillation 
Spectrometry, 2008, p. 13-18; (c) M. Hamel, S. Normand and V. Simic PCT Patent application WO2009095376, 2009.

[14] N. Divani-Vais, E. Bayat, M. M. Firoozabadi and N. Ghal-Eh, Radiat Prot. Dosim., 2013, 154, 381-384.

[15] J. Shi, M. Huang, Y. Xin, Z. Chen, Q. Gong, S. Xu and S. Cao, Mater Lett., 2005, 59, 2199-2203.
[16] G. H. V. Bertrand, J. Dumazert, F. Sguerra, R. Coulon, G. Corre and M. Hamel, J. Mater. Chem. C 2015, 3, 6006-6011.

[17] http://faster.in2p3.fr/ 


\section{Entry for the Table of Contents}

\section{FULL PAPER}

We report herein the synthesis of $\mathrm{N}$-(2-ethylhexyl)carbazole and its use as a monomolecular liquid scintillator.

Author(s), Corresponding Author(s)*

Page No. - Page No.

Title

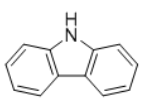

$\underset{\mathrm{BMF}, \mathrm{RT}, 16 \mathrm{~h}}{\stackrel{\mathrm{NaH}}{\longrightarrow}}$

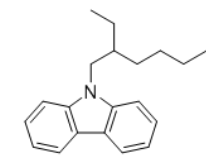

Scintillator

\section{yield $82 \%$}

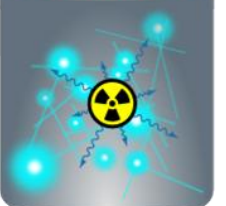

